\title{
Thirteen polymorphic microsatellite markers for the European green toad Bufo viridis viridis, a declining amphibian species
}

\author{
Christophe Dufresnes - Caroline Betto-Colliard • \\ Nicolas Perrin • Matthias Stöck
}

Received: 26 October 2010/Accepted: 1 November 2010

(C) Springer Science+Business Media B.V. 2010

\begin{abstract}
We report 13 new polymorphic microsatellite markers for the European green toad Bufo viridis viridis (B. viridis subgroup), a declining amphibian from Central, Southeastern and Eastern Europe. Diversity at these loci estimated for 19 individuals ranged from two to ten alleles. Most of these primers also cross-amplify in related WestMediterranean green toad species (Bufo balearicus, B. siculus and $B$. boulengeri). These microsatellites will be useful for conservation genetics of threatened Bufo viridis viridis populations and evolutionary studies of green toad taxa in secondary contact to examine hybridization.
\end{abstract}

Keywords Bufo viridis viridis · Polymorphic

microsatellites · Population genetics ·

Conservation research

\section{Introduction}

Green toads are widespread in the Western Palearctics (Stöck et al. 2006). They comprise a monophyletic

C. Dufresnes · C. Betto-Colliard · N. Perrin · M. Stöck ( () Department of Ecology and Evolution, University of Lausanne, Biophore, 1015 Lausanne, Switzerland

e-mail: matthias.stoeck@unil.ch

C. Dufresnes

e-mail: christophe.dufresnes@unil.ch

C. Betto-Colliard

e-mail: caroline.betto-colliard@unil.ch

N. Perrin

e-mail: nicolas.perrin@unil.ch radiation of at least twelve major haplotype groups, several of which occur in Europe (for overview: Stöck et al. 2008 incl. refs.). The mitochondrial haplotype group that occurs at the type locality (Vienna) of Bufo viridis viridis (Laurenti 1768), is distributed from Crete, across Western Greece, West of the Carpathians into Central Europe, where it reaches its most Western range border approximately at the Rhine River. Another range part lies in Eastern Russia and reaches further East into northern Kazakhstan. Several taxa of the B. viridis subgroup are under threat including the Central European taxon $B$. viridis viridis (Podloucky and Manzke 2003), and the lineage distributed around the Baltic Sea, B. variabilis. At their Western range part both are considered rare and "threatened by extinction" or "strongly threatened", and even "extinct" in Switzerland (Grossenbacher 2003). This threat resulted in European legislation, and today "green toads" are generally protected according to Appendix 2 of the Berne Convention (1979), so far ratified in 45 countries, and according to Appendix IV of the Habitats Directive (1992). Species protection plans have been or are going to be implemented (e.g. Plan National 2010; ref. in Stöck et al. 2008). Besides major threats from industrial agriculture, traffic and its infrastructure, general habitat destruction and the potential threat from chytridomycosis (e.g. Kilpatrick et al. 2010), habitat fragmentation plays a role. As anurans that react opportunistically to changing breeding conditions, their great mobility requires connected habitats for viable metapopulations. Protection should be accompanied by research designed to test efficiency of measurements (e.g. population genetics). Microsatellites have so far been published for Western Mediterranean and Central Asian green toads (Colliard et al. 2009; Stöck et al. 2010), but they have not been available for Bufo viridis viridis. 
Table 1 Thirteen new microsatellites for Bufo viridis viridis

\begin{tabular}{|c|c|c|c|c|c|c|c|c|c|}
\hline Locus & GenBank & Ised label & Primer sequences & Repeat motif & $\mathrm{Na}$ & $\begin{array}{l}\text { Allele size range } \\
\text { (bp) }\end{array}$ & $\begin{array}{l}\text { Sampling } \\
\text { locality }\end{array}$ & Ho & $\mathrm{He}$ \\
\hline $\operatorname{cal} \mu 10$ & AF2C & FAM & $\begin{array}{l}\text { F: ATGCTTTCATTGCTGGAGAGG } \\
\text { R: GCCСССТTCCСTGTACCAAG }\end{array}$ & $(\mathrm{AG})_{\mathrm{n}}$ & 2 & 56 & $\begin{array}{l}\text { Santa Croce } \\
\text { Cusignana }\end{array}$ & $\begin{array}{l}0.14 \\
0.00\end{array}$ & $\begin{array}{l}0.14 \\
\mathrm{NC}\end{array}$ \\
\hline aturaC124 & FJ613130 & HEX & $\begin{array}{l}\text { F: CCCCATAACAAACTGCTGATA } \\
\text { R: CTTGCTACAGGGCACTACAAC }\end{array}$ & $(\mathrm{TACA})_{\mathrm{n}}$ & 2 & $160-163$ & $\begin{array}{l}\text { Santa Croce } \\
\text { Cusignana }\end{array}$ & $\begin{array}{l}0.29 \\
0.00\end{array}$ & $\begin{array}{l}0.44 \\
\mathrm{NC}\end{array}$ \\
\hline aturaC107 & FJ613131 & FAM & $\begin{array}{l}\text { F: ACGGTGTGGATTCCTTTAG } \\
\text { R: AGCAGCATACAACAGGTGA }\end{array}$ & $(\mathrm{TACA})_{\mathrm{n}}$ & 2 & $223-227$ & $\begin{array}{l}\text { Santa Croce } \\
\text { Cusignana }\end{array}$ & $\begin{array}{l}0.00 \\
0.42\end{array}$ & $\begin{array}{l}\mathrm{NC} \\
0.52\end{array}$ \\
\hline aturaC218 & FJ61 & ATTO & $\begin{array}{l}\text { F: GCATACCTTGGTCAATGATG } \\
\text { R: CAGGGAGAGCAGGCTTTA }\end{array}$ & $(\mathrm{TAGA})_{\mathrm{n}}$ & 10 & 15 & $\begin{array}{l}\text { Santa Croce } \\
\text { Cusignana }\end{array}$ & $\begin{array}{l}1.00 \\
1.00\end{array}$ & $\begin{array}{l}0.91 \\
0.83^{*}\end{array}$ \\
\hline aturaC201 & HQ3 & HEX & $\begin{array}{l}\text { F: AGGACCCAGGATTTCCAT } \\
\text { R: GCTTCTACCAAAGACTGTTCC }\end{array}$ & $(\mathrm{TCTA})_{\mathrm{n}}$ & 8 & 9 & $\begin{array}{l}\text { Santa Croce } \\
\text { Cusignana }\end{array}$ & $\begin{array}{l}0.71 \\
0.75\end{array}$ & $\begin{array}{l}0.86 \\
0.77\end{array}$ \\
\hline aturaC223 & FJ61? & ATTO & $\begin{array}{l}\text { F: CAGAGGTCAAGAGGGAGAAG } \\
\text { R: GGCACCACATCCTGATTAG }\end{array}$ & $(\mathrm{TCTA})_{\mathrm{n}}$ & 4 & 17 & $\begin{array}{l}\text { Santa Croce } \\
\text { Cusignana }\end{array}$ & $\begin{array}{l}0.57 \\
0.42\end{array}$ & $\begin{array}{l}0.58 \\
0.42\end{array}$ \\
\hline aturaC203 & FJ613132 & HEX & $\begin{array}{l}\text { F: TTGAGGTCCACTAACCACTGTA } \\
\text { R: AGGATTGCCTGAAAACATTTAC }\end{array}$ & $(\mathrm{TAGA})_{\mathrm{n}}$ & 7 & $201-227$ & $\begin{array}{l}\text { Santa Croce } \\
\text { Cusignana }\end{array}$ & $\begin{array}{l}0.86 \\
1.00\end{array}$ & $\begin{array}{l}0.87 \\
0.82\end{array}$ \\
\hline BaturaC224 & HQ386138 & ATTO & $\begin{array}{l}\text { F: TTTGGAAGGAAAGCATCTG } \\
\text { R: TCATCAACCTGGGATAATACTG }\end{array}$ & $\begin{array}{l}(\mathrm{TAGA})_{\mathrm{n}} \\
(\text { TAATA })_{\mathrm{n}}\end{array}$ & 10 & $161-213$ & $\begin{array}{l}\text { Santa Croce } \\
\text { Cusignana }\end{array}$ & $\begin{array}{l}0.86 \\
0.92\end{array}$ & $\begin{array}{l}0.80 \\
0.88\end{array}$ \\
\hline BaturaD105 & FJ613136 & FAM & $\begin{array}{l}\text { F: AATGCAGAGATGCCATGTG } \\
\text { R: TTGGATGATCTTCCTTTCAAAG }\end{array}$ & $(\mathrm{TAGA})_{\mathrm{n}}$ & 5 & $182-207$ & $\begin{array}{l}\text { Santa Croce } \\
\text { Cusignana }\end{array}$ & $\begin{array}{l}0.29 \\
1.00\end{array}$ & $\begin{array}{l}0.47 \\
0.76\end{array}$ \\
\hline BaturaD210 & HQ386139 & FAM & $\begin{array}{l}\text { F: GGCCAATGAAAGAGGAAC } \\
\text { R: GCTTCGCTTATCCCTAGTTAC }\end{array}$ & $(\mathrm{TACA})_{\mathrm{n}}$ & 2 & $174-178$ & $\begin{array}{l}\text { Santa Croce } \\
\text { Cusignana }\end{array}$ & $\begin{array}{l}0.14 \\
0.08\end{array}$ & $\begin{array}{l}0.14 \\
0.08\end{array}$ \\
\hline BaturaD5 & FJ613138 & HEX & $\begin{array}{l}\text { F: CCTCTTTTACCTCTGACAGTGC } \\
\text { R: ACAGGCATGAAAACCATTGA }\end{array}$ & $(\text { TCTA })_{n}$ & 3 & $113-152$ & $\begin{array}{l}\text { Santa Croce } \\
\text { Cusignana }\end{array}$ & $\begin{array}{l}0.50 \\
0.36\end{array}$ & $\begin{array}{l}0.62 \\
0.31\end{array}$ \\
\hline BaturaD106 & FJ613137 & FAM & $\begin{array}{l}\text { F: CCATTCCGAAGATGATAACA } \\
\text { R: CACGCAAAAAATACAGATGAC }\end{array}$ & $(\mathrm{GATA})_{\mathrm{n}}$ & 4 & $210-224$ & $\begin{array}{l}\text { Santa Croce } \\
\text { Cusignana }\end{array}$ & $\begin{array}{l}0.43 \\
0.58\end{array}$ & $\begin{array}{l}0.36 \\
0.63\end{array}$ \\
\hline BaturaC205 & FJ613133 & HEX & $\begin{array}{l}\text { F: TCATCATCATCCСTCTCTATG } \\
\text { R: CCAAAGCAAAGTCAGTAACAG }\end{array}$ & $(\text { TCTA })_{\mathrm{n}}$ & 6 & $172-256$ & $\begin{array}{l}\text { Santa Croce } \\
\text { Cusignana }\end{array}$ & $\begin{array}{l}0.86 \\
0.91\end{array}$ & $\begin{array}{l}0.79 \\
0.72\end{array}$ \\
\hline
\end{tabular}

GenBank accession number, Used label ABI-compatible fluorescent dye (forward primer labelled), Ho observed heterozygosity, He expected heterozygosity, $N C$ He not calculated when $\mathrm{Ho}=0$

* Locus significantly deviates from Hardy-Weinberg expectations after Bonferroni correction

\section{Materials and methods}

As reported previously (Colliard et al. 2009), microsatellite markers were obtained from a genomic library produced by the Genetic Identification Services, (www.genetic-idservices.com, DNA from Bufo baturae, voucher: ZMB 58770, Pasu, Karakoram, $36^{\circ} 29^{\prime} 20.0^{\prime \prime} \mathrm{N}, 74^{\circ} 52^{\prime} 47.6^{\prime \prime} \mathrm{E}$, a related species from Pakistan). We also tested one primer pair, developed originally for Bufo calamita (Rowe et al. 2000) that has successfully been used in other green toads.

Genomic DNA was extracted from ethanol preserved muscle, tail tips (tadpoles) and frozen buccal swabs (QIAGEN DNeasy kit). Primers were tested by performing polymerase chain reactions (PCR) in $10 \mu \mathrm{l}$ reactions, containing $1 \mu \mathrm{l}$ DNA (10 ng/ $\mu \mathrm{l}), 1 \mu \mathrm{l} 10 \times$ PCR buffer $\left(15 \mathrm{mM} \mathrm{MgCl}_{2}\right), 0.2 \mu \mathrm{l}$ dNTPs $(25 \mathrm{mM}), 0.2 \mu \mathrm{l}$ each primer $(10 \mu \mathrm{M})$; forward fluorescently labeled), $0.2 \mu \mathrm{l}$ additional $\mathrm{MgCl}_{2}(25 \mathrm{mM})$, and $0.1 \mu \mathrm{l}$ QIAGEN Taq (5 U/ $\mu \mathrm{l})$. Amplifications were carried out on GeneAmp PCR System $9700\left(\mathrm{ABI} ; 3 \mathrm{~min}\right.$ at $94^{\circ} \mathrm{C}, 38 \times\left(1 \mathrm{~min}\right.$ at $94^{\circ} \mathrm{C}$, $1 \mathrm{~min}$ at $50^{\circ} \mathrm{C}, 1 \mathrm{~min}$ at $\left.72^{\circ} \mathrm{C}\right), 4 \mathrm{~min}$ at $72^{\circ} \mathrm{C}$ ). Fragment analyses were performed (ABI PRISM 3100 sequencer) and allele sizes scored using the size standard ROX-350 (GeneMapper 4.0, AppliedBiosystems, Inc.).

To assess variability and genetic diversity in natural populations, we genotyped 19 Bufo v. viridis from two neighboring localities in northern Italy (Santa Croce, $45^{\circ} 51^{\prime} 0.32^{\prime \prime} \mathrm{N}, 12^{\circ} 9^{\prime} 4.02^{\prime \prime} \mathrm{E}, \mathrm{n}=7$; Cusignana, $45^{\circ} 47^{\prime}$ $0.35^{\prime \prime} \mathrm{N}, 12^{\circ} 11^{\prime} 38.98^{\prime \prime} \mathrm{E}, \mathrm{n}=12$; no larger sample size was available). Sequencing of the mtDNA d-loop (data not shown) showed our test populations to exhibit no signs of introgression from $B$. balearicus whose range borders to the south of the Po valley (Colliard et al. 2010). All 13 loci were tested for polymorphisms and deviations from 
Hardy-Weinberg equilibrium using Arlequin 3.11 (Excoffier et al. 2005). We also calculated differentiation (Fst) and inbreeding (Fis) coefficients (Arlequin), tested for linkage disequilibrium (Fstat; Goudet 1995) and checked for null alleles (Micro-Checker 2.2.3; van Oosterhout et al. 2004).

\section{Results}

Thirteen microsatellites (Table 1) could be successfully amplified and turned out to be polymorphic in Bufo $v$. viridis from the two test populations. Ten of them have been shown to cross-amplify in the related species Bufo balearicus, B. siculus, and B. boulengeri (for details: Colliard et al. 2009).

Our two test populations were significantly differentiated (Fst: $0.104, P<0.01$ ). However, inbreeding coefficients (Fis) were not significant and all loci but one (C218 in Cusignana) met Hardy-Weinberg expectations in both sampling areas. Furthermore, we neither detected evidence for null alleles, large allelic dropout, or scoring errors due to stuttering. Allele numbers ranged from 2 to 10, with some loci being highly polymorphic (allelic diversity $\mathrm{Na}>6)$ as in related species (C205, C218; Colliard et al. 2009). We did not detect significant linkage disequilibrium between our loci in any population after Bonferroni corrections.

The microsatellites primers presented here will be useful to assess regional genetic structures of Bufo viridis viridis populations for conservation and other purposes. In addition, these markers will also be applicable to conduct population genetic studies in contact zones involving Bufo viridis and related taxa (e.g. B. variabilis.)

Acknowledgments We thank Lucio Bonato, Nicola Novarini, Stefano Mazzotti, and G. Francesco Ficetola for providing samples that served, in part, to choose pure $B$. viridis viridis populations examined in this paper. This study was in part supported by a grant from the "Fondation 450ème anniversaire" of the University of Lausanne (November 23, 2009 to M.St.).

\section{References}

Berne Convention (1979) Bern convention on the conservation of European wildlife and natural habitats 1979, also known as the Bern convention, came into force on 1 June 1982, (http:// conventions.coe.int/treaty/en/Treaties/Html/104.htm)
Colliard C, Sicilia A, Moritz C, Perrin N, Stöck M (2009) New polymorphic microsatellite markers and development of mitotyping primers for West Mediterranean green toad species (Bufo viridis subgroup). Mol Ecol Resour 9(4):1138-1140

Colliard C, Sicilia A, Turrisi GF, Arculeo M, Perrin N, Stöck M (2010) Strong reproductive barriers in a narrow hybrid zone of West-Mediterranean green toads (Bufo viridis subgroup) with Plio-Pleistocene divergence. BMC Evol Biol 10:232

Excoffier L, Laval G, Schneider S (2005) Arlequin (version 3.0): an integrated software package for population genetics data analysis. Evol Bioinform 1:47-50

Goudet J (1995) Fstat (version 1.2): a computer program to calculate F-statistics. J Hered 86:485-486; program available: http:// www2.unil.ch/popgen/softwares/fstat.htm

Grossenbacher K (2003) Die Wechselkröte (Bufo viridis) in der Schweiz: historische und aktuelle Situation. Mertensiella 14: $147-152$

Habitats directive (1992) Habitats directive — council directive 92/43/EEC on the conservation of natural habitats and of wild fauna and flora. http://ec.europa.eu/environment/nature/legislation/habitatsdirective/ index_en.htm

Kilpatrick C, Briggs J, Daszak P (2010) The ecology and impact of chytridiomycosis: an emerging disease of amphibians. Trends Ecol Evol 25(2):109-118

Plan National (2010) Plan national d'action crapaud vert (pour La France). DREAL Lorraine-BIOTOPE Draft: Août 2010; see: http://www.lorraine.developpement-durable.gouv.fr/IMG/pdf/ cahier_des_charges_precis_cle2deb1d.pdf

Podloucky R, Manzke U (2003) Verbreitung, Ökologie und Schutz der Wechselkröte (Bufo viridis). DGHT Rheinbach, Mertensiella 14:1-327

Rowe G, Beebee T, Burke T (2000) A further four polymorphic microsatellite loci in the natterjack toad Bufo calamita. Conserv Genet 1:371-372

Stöck M, Moritz C, Hickerson M, Frynta D, Dujsebayeva T, Eremchenko V, Macey JR, Papenfuss TJ, Wake DB (2006) Evolution of mitochondrial relationships and biogeography of Palearctic green toads (Bufo viridis subgroup) with insights in their genomic plasticity. Mol Phylogenet Evol 41:663-689

Stöck M, Roth P, Podloucky R, Grossenbacher K (2008) Wechselkröten - unter Berücksichtigung von Bufo viridis virdis Laurenti, 1768; Bufo variabilis (Pallas, 1769); Bufo boulengeri Lataste, 1879; Bufo balearicus Böttger, 1880 und Bufo siculus Stöck, Sicilia, Belfiore, Lo Brutto, Lo Valvo und Arculeo, 2008. In: Grossenbacher K (ed) Handbuch der Amphibien und Reptilien Europas. vol. 5 (Froschlurche II) [Handbook of the Amphibians and Reptiles of Europe. vol. 5 (Anura II)]. AULA-Verlag, Wiesbaden (invited book chapter), pp. 413-498

Stöck M, Ustinova J, Lamatsch D, Schartl M, Perrin N, Moritz C (2010) A vertebrate reproductive system involving three ploidy levels: hybrid origin of triploids in a contact zone of diploid and tetraploid Palearctic green toads (Bufo viridis subgroup). Evolution 64(4):944-959

Van Oosterhout C, Hutchinson WF, Wills DPM, Shipley P (2004) MICRO-CHECKER: software for identifying and correcting genotyping errors in microsatellite data. Mol Ecol Notes 4: 535-538 